\title{
The Mere Exposure Effect Is Sensitive to Color Information
} Evidence for Color Effects in a Perceptual Implicit
Memory Test

\author{
Almut Hupbach, ${ }^{1}$ André Melzer, ${ }^{2}$ and Oliver Hardt ${ }^{1}$ \\ ${ }^{1}$ The University of Arizona, Tucson, United States \\ ${ }^{2}$ University of Lübeck, Germany
}

\begin{abstract}
Priming effects in perceptual tests of implicit memory are assumed to be perceptually specific. Surprisingly, changing object colors from study to test did not diminish priming in most previous studies. However, these studies used implicit tests that are based on object identification, which mainly depends on the analysis of the object shape and therefore operates color-independently. The present study shows that color effects can be found in perceptual implicit tests when the test task requires the processing of color information. In Experiment 1, reliable color priming was found in a mere exposure design (preference test). In Experiment 2, the preference test was contrasted with a conceptually driven color-choice test. Altering the shape of object from study to test resulted in significant priming in the color-choice test but eliminated priming in the preference test. Preference judgments thus largely depend on perceptual processes. In Experiment 3, the preference and the color-choice test were studied under explicit test instructions. Differences in reaction times between the implicit and the explicit test suggest that the implicit test results were not an artifact of explicit retrieval attempts. In contrast with previous assumptions, it is therefore concluded that color is part of the representation that mediates perceptual priming.
\end{abstract}

Keywords: implicit memory, color memory, perceptual implicit processes, mere exposure effect

Memory can be assessed explicitly by asking participants to recall or recognize prior events or implicitly by engaging participants in apparently unrelated cognitive tasks that contain previously studied and unstudied stimuli. An implicit effect (priming effect) is revealed by an enhanced performance for studied stimuli compared with unstudied stimuli. For example, pictures that were part of a study session are named faster in a subsequent picture-naming test than are previously unstudied pictures (e.g., Cave, Bost, \& Cobb, 1996).

Memory tests have been classified as either conceptual or perceptual according to their main underlying processes (for a review, see Roediger \& McDermott, 1993). Memory tests that rely on perceptual processing of the stimuli are therefore classified as perceptual tests, whereas tests that operate on semantic aspects of the stimuli (e.g., meaning) are classified as conceptual tests. This classification is not meant as a strict dichotomy. Instead, most memory tests rely on both processes with different weighting (e.g., Craik, Moscovitch, \& McDowd, 1994; Weldon, 1991). Memory performance depends on how much the processes evoked by the test overlap or recapitulate the processes that were engaged during encoding (transfer-appropriate processing [TAP] principle, e.g., Roediger, 1990; Roediger, Weldon, \& Challis, 1989). Effects in perceptual implicit tests (perceptual priming) are assumed to be perceptually specific- that is, they depend on the exact repetition of those perceptual processes at test that were induced by the perceptual features of the stimuli at study. Accordingly, it has been shown that perceptual priming is diminished when study and test sessions differ in modality (e.g., Jacoby \& Dallas, 1981; Roediger \& Blaxton, 1987), stimulus presentation mode (from pictures to words or vice versa, e.g., Rajaram \& Roediger, 1993; Srinivas, 1993), or object shape (by presenting different pictures of the same-named objects, Cave et al., 1996).

Contrary to the perceptual specificity assumption, attempts to find color-specific priming effects have produced inconsistent results. Changes in item color between study and test did not affect object naming (Cave et al., 1996; Lloyd-Jones, 2005, Experiment 1; Vernon \& Lloyd-Jones, 2003, Experiments 1 and 2a), perceptual facilitation in object drawing (Treisman, 1992), picture fragment identification (Wippich \& Mecklenbräuker, 1998), word-picture matching (Zimmer \& Steiner, 2003), or object preference (Seamon et al., 1997). ${ }^{1}$ Yet color specificity was found for color naming (Musen \& O’Neill, 1997; Musen, Szerlip, \& Szerlip, 1999). Irrespective of the fate of color in implicit tests, color information was explicitly remembered well above chance levels in most studies, thus indicating that item color was stored in memory.

How can the apparent color insensitivity of perceptual 
implicit tests be explained? Cave et al. (1996) pointed out that almost every test that has been used to assess color effects depends on processes of visual object identification. Basic-level object identification relies on the object shape that is largely defined by the object edges (e.g., "geons," Biederman \& Ju, 1988). In most circumstances, object edges are accessible regardless of actual size, left-right orientation, and color variations. Cave et al. concluded "that repetition priming can be insensitive to the same stimulus characteristics as basic object identification is" (p. 650). In line with this argument, it has been shown that, like changing color from study to test, changing size, left-right orientation (Cooper, Schacter, Ballesteros, \& Moore, 1992), location (Treisman, 1992), and fill pattern (Cave et al.) does not affect priming in identification paradigms.

A similar explanation was suggested by Seamon et al. (1997), who found that changes of size, left-right orientation, or color had no influence on the mere exposure effect in an object preference task. The mere exposure effect refers to the phenomenon in which repeated unreinforced exposure to an item increases the probability that it will be preferred over new items in judgments of liking. According to Seamon et al. (1997; see also Seamon \& Delgado, 1999), preference judgments, like other visual object priming tests (e.g., identification paradigms), reflect the operation of the Structural Description System, a subsystem of the Perceptual Representation System (PRS, e.g., Schacter, 1992, 1994). This system captures the global shape and structure of an object by representing the spatial relations among the object's parts, but does not compute variable surface features such as size, orientation, and color. In contrast, explicit object memory is assumed to rely on a different, episodic memory system that captures spatial, temporal, contextual, and semantic information about objects. The view that preference judgments are based on a different representational system than performance in explicit memory tests is controversial. Lawson (2004) found similar effects of depth rotation and mirror reflection on recognition and preference tests. In accordance with processing accounts of implicit memory, she concludes that "the same view-sensitive representations underlie object recognition across a variety of tasks (including both explicit and implicit memory tasks)" (Lawson, 2004, p. 1180). Participants may use different processing strategies in implicit and explicit memory tasks.

We think that assumptions about the nature of the representations underlying perceptual priming are still quite speculative. With regard to the failure to observe colorspecific priming effects, one could argue that the selected study and test tasks "by mischance" evoke cognitive processes that operate color independently. Color might be part of the representation underlying perceptual priming, and what needs to be found is simply the right cue or process to access that information (cf. Wippich, Mecklenbräuker, \& Baumann, 1994). It was acknowledged by Cave et al. (1996) and experimentally shown by Srinivas (1996) that the system mediating perceptual priming does indeed operate more flexibly than initially thought. For instance, Srinivas found effects of size changes on perceptual priming when both the study and test task required the pro- cessing of size information. Specifically, in both the study and the test session, drawings of two different sizes were presented. Participants were asked to decide whether the depicted objects were larger or smaller than a reference object (for example, an ordinary chair). In the test session, participants responded faster to previously studied drawings than to new ones. This overall priming effect was significantly diminished when the size of the drawings was changed between study and test, thus indicating size specificity. In contrast to size effects, however, changes in leftright orientation did not affect priming, even when the study and test task encouraged the processing of left-right orientation. Srinivas concluded that size, but not orientation information, can be optionally included in the PRS. Although her study clearly shows that certain features might affect perceptual priming when the task requires the processing of those features, there is no method to predict a priori which perceptual feature might be utilized by the system. Srinivas speculated that size can be optionally represented because size, but not left-right orientation, is sometimes useful in discriminating between objects. This could also hold for color information because color can be a powerful feature in visual object discrimination (e.g., when differentiating an edible mushroom from a toadstool or when stopping at a red traffic light).

Recently, Lloyd-Jones (2005) and Vernon and LloydJones (2003) followed Srinivas's (1996) line of arguments in the discussion of a series of experiments with diagnostically colored natural objects (e.g., yellow banana). When the implicit test was object naming, changes in color from study to test (e.g., purple banana) did not influence priming. Yet, when participants decided whether each test stimulus was a real object on the basis of its color (i.e., coloredobject decision), priming was found only for objects that remained the same color. The authors attributed the finding of color specificity in the latter test as reflecting cognitive flexibility in the use of information by implicit memorial systems. This flexibility is thought to be driven by the extent to which the perceptual feature (i.e., color) is useful in differentiating between different objects. In the case of colored-object decision, the retrieval task requires the reactivation of both object and color information, and thus color constancy aids implicit performance. Object naming, on the other hand, only requires discriminating between different objects - a basic-level process that relies solely on reactivation of stored shape-based information. Although the latter test is undoubtedly a perceptual test, colored-object decision may not readily be characterized in terms of its underlying cognitive mechanisms. Actually, Vernon and Lloyd-Jones state that they "remain neutral as to whether stored object color knowledge contributing to priming in colored-object decisions is perceptual or conceptual" (p. 799).

In sum, most studies on perceptual implicit color effects so far have lacked appropriate retrieval tasks that required the processing of color information. As Srinivas (1996) has shown for size information, the present study asks whether color effects can be obtained in perceptual priming tests when the test task requires judgments that are based on color processing. Results from this study may allow us to 
determine whether the representational system mediating perceptual priming utilizes color information.

\section{Experiment 1}

In the present study, we studied color effects with a mere exposure design. The mere exposure effect was chosen because the most prominent paradigm that is used to demonstrate the phenomenon - the preference test-seemed to be especially well-suited for studying color effects independently of shape-based priming effects. Seamon et al. (1997) did not find that color changes moderate the mere exposure effect. Participants in their study always had to choose between two items representing different (old and new) objects at test. Color status was varied between subjects; that is, for half of the participants, the color of the studied items was changed, and for the other half, the color remained constant during study and test. In order to reveal implicit color effects, participants in the constant-color group would have had to prefer old objects over new objects significantly more often than participants in the changed-color group. This was not the case. It might be possible, however, to find color effects in preference tests when these effects are not indirectly calculated as diminished object priming effects. Although Seamon et al.'s approach is in line with studies on perceptual effects in identification paradigms, it seems to be a rather conservative test of implicit color effects. In the present study, implicit color effects were thus assessed more directly. Participants had to choose between two (Experiment 1A) or four (Experiments $1 \mathrm{~B}$ and 2 ) versions of drawings of the same object, versions that differed only in terms of color. Therefore, the preference judgments were solely based on color information. Additionally, following Srinivas's (1996) findings on the methodological prerequisites for size effects, color processing was encouraged in both the study and the test session. In the study session, participants were asked to judge how well certain colors fit certain objects. In the test session, participants were asked which one of two differently colored drawings of the same object they preferred. Therefore, both the study and the test task explicitly required the processing of color information.

\section{Experiment 1A}

\section{Method}

\section{Design and Participants}

A one-factorial design was used. The only variable that was manipulated within subjects was the item status at test. Half of the items were repeated from the study session ("old"), whereas the other half of the items were first shown at test ("new"). Twenty-six adults participated in the experiment. The participants were undergraduate students at the University of Trier (20 females, 6 males; mean age $=23$ years; range $=19$ to 32 years). They received course credit for their participation.

\section{Materials}

Two lists of 36 target items were constructed. The items were presented as line drawings (single-colored outlines presented on a black computer screen; see Figure 1). The items depicted everyday objects that may occur in a variety of equally plausible colors (e.g., cars, pens) rather than being confined to some typical color. Therefore, all of the colors in which these objects were displayed in this experiment (i.e., blue, green, yellow, and red) were of approximately the same typicality. Additionally, each list contained three filler items at the beginning and the end of the list to compensate for primacy and recency effects, respectively. Participants received one of the lists (study list) during the study session; the other list served as a new list at test. Across participants, each list served equally often as a study list and as a new list. During the test session, both lists were combined. Thus, each item was used as an old or a new one with equal frequency.

Only one item was presented at a time during study. In both study lists there were nine items for each of the four colors. Each object was randomly assigned one of the four colors. On each trial of the preference test, drawings were simultaneously displayed in two color versions and were located side by side on the computer monitor. The only perceptual difference between the two drawings was the color in which they were presented. One of the drawings showed the object in the same color in which it was presented in the study session, and the other drawing showed the object in a different color. This means that for all old items, one of the drawings shown was the original drawing in the color that participants had seen during the study session. The drawing presenting the object in the studied color appeared equally often on the left and right side of the screen.

For new items, two colored versions of the same drawing were presented side by side, as for old items. One of the colored versions was a priori designated to be the target color (see list construction noted previously).

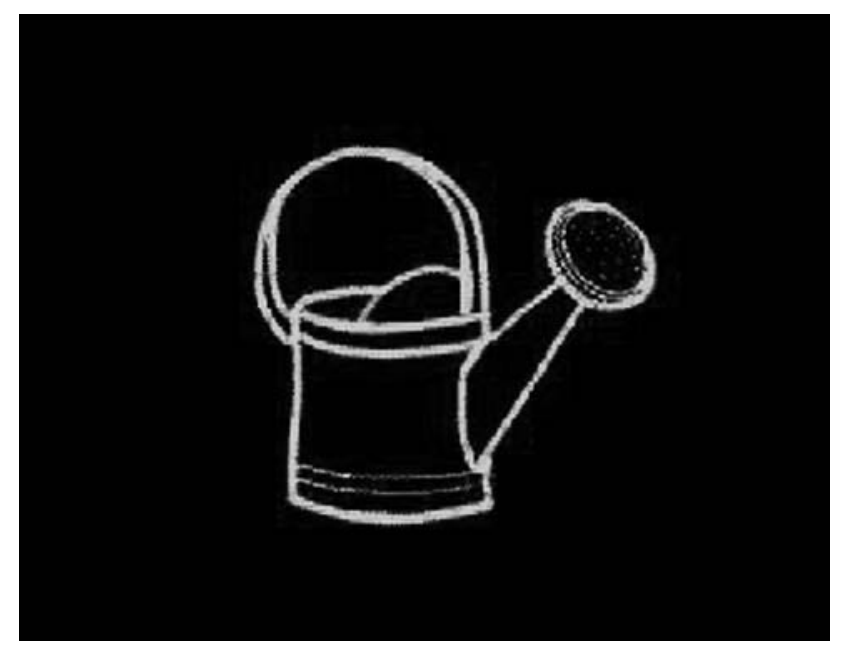

Figure 1. Example of a line drawing used as target item. Note: Items were presented in one of four different colors (red, green, yellow, blue). 
Throughout the experiment, the items were presented in different quasi-random orders such that the same color or the same type of test item (old or new) could follow each other only once (this restriction applies to all experiments in the present study).

\section{Procedure}

Participants were tested in a computer lab with four computer desks separated by movable office walls. Participants used headphones throughout the experiment to minimize distractive noise. The instructions for both the study and the test task were presented on the computer screen and informed participants that they were participating in a normative study on how well certain colors fit certain objects. Participants were asked in the study session to rate how well each presented drawing fit its color. Four rating buttons (good, rather good, rather bad, bad) were presented side by side under the presented line drawing. Participants rated each drawing by clicking on one of the buttons. They were not informed about a later memory test for the drawings (incidental learning). After the participants had read the instructions for the study session, the items of one of the study lists (36 items) were presented one at a time. Immediately after study, the test session began. On each trial, two color versions of the same drawing were simultaneously shown side by side, and the participants were asked to choose the preferred drawing by moving the mouse and clicking directly on the drawing. The starting position of the mouse cursor was constant for every trial (center of the screen). Participants were told that there were no right or wrong answers, and that they should answer quickly according to their first impression. Seventy-two item pairs, made up of items from the study list and the new list, were presented. It took about twenty minutes to complete the entire experiment.

\section{Results}

The significance level for all the results of this and the following experiments was set at $\alpha=.05$. Results reported as marginally significant were in the range of .10 $\geq \alpha>.05$. Results were analyzed by participant and by item. For the by-participant analyses the data were pooled for each participant over the different items in each condition. For the by-item analyses the data were pooled over the different participants who were presented with that item in a given condition. Results of the by-participant analyses are marked with subscript " 1 ," and results of the by-item analyses bear subscript "2."

The percentage of drawings chosen in the target color for old and new items in the implicit preference test was analyzed with a paired-samples $t$-test. Participants selected target colors significantly more often for old items $(M=$ $56.4 \%, S E=11.4 \%$ ) than for new items $[M=49.3 \%, S E$ $=8.7 \%, t_{1}(25)=2.47, p_{1}=.01, t_{2}(71)=3.31, p_{2}<.01$, one-tailed]. Target-color selection for new items was not different from chance (i.e., $50 \%, t<1$ ).

The mean response times for old and new items were analyzed with a paired-samples $t$-test. Participants re- sponded faster to old items $(M=.85 \mathrm{~s}, S E=.17 \mathrm{~s})$ than to new items $\left[M=.89 \mathrm{~s}, S E=.15 \mathrm{~s}, t_{1}(25)=1.94, p_{1}=\right.$ .03 , one-tailed].

\section{Discussion}

Experiment 1A showed a significant implicit color effect in a preference test in which participants had to choose between two identical drawings that differed only in color (same versus different, as in the study list). This means that preference judgments were based solely on color information. To our knowledge, this is the first demonstration of implicit color effects in a preference task. Our result is inconsistent with Seamon et al.'s (1997) finding that the mere exposure effect is insensitive to color information. In their study, participants always had to choose between two different objects (old and new). For half of the participants, the color of the old objects remained constant, and for the other half, it was changed from study to test. In both groups, however, all objects were shown in the same color during the test session. This means that the presented objects only differed in their global shape. Additionally, although it can be assumed that all participants in the colorchange group noticed the change in color, presenting objects in the same color might have encouraged people to ignore the color change and to focus on the object shape. Human visual-information processing is known to be tuned to noticing visual changes, not constancy of features. Therefore, it is likely that Seamon et al.'s (1997) test procedures especially supported priming of shape-related processes (i.e., focusing on the feature that was actually changing). In light of Srinivas's (1996) findings that size information only affected priming effects when the processing of size information was relevant for both the study and the test session, Seamon et al. (1997) seem to have created test conditions that especially disfavored color priming, because neither at study nor at test was color information salient or required to process.

We tried to replicate the color priming effect found in Experiment 1A under slightly different test conditions in Experiment $1 \mathrm{~B}$ to ensure that the effect is a stable one.

\section{Experiment 1B}

The main purpose of Experiment 1B was to replicate the significant color priming effect we found in Experiment $1 \mathrm{~A}$. Because the effect was rather small, we included more participants $(N=48)$ to enhance power of our measurement. In contrast with Experiment 1A, we always presented four drawings simultaneously at test. These four drawings differed only in color (red, green, blue, yellow). Hence, baseline performance (i.e., selecting the target color to new items) was expected to be $25 \%$. This test format was used to increase response alternatives. This made it more likely that one of the presented colors was the color the participant preferred for a given object. Additionally, we were interested in whether the color priming effect found in Experiment 1A is a very short-lived phenomenon. 
In order to prolong the retention interval in Experiment 1B, we inserted a filler task between the study and the test session.

\section{Method}

\section{Design and Participants}

We used the same design as in Experiment 1A. Forty-eight adults who did not take part in Experiment 1A participated in the experiment. The participants were undergraduate students at the University of Arizona (24 females, 24 males; mean age $=19$ years; range $=18$ years to 21 years) who received course credit for their participation.

\section{Material and Procedure}

The two study lists of Experiment 1A were shortened by four items each (i.e., 32 items in each list). This was done because a filler task was added. The material was otherwise identical to Experiment 1A. The procedure for the study and test session was identical to Experiment 1A with the following exceptions:

1. We included a filler task. A 15- to 20-minute-long picture fragment completion test was administered between the study and the test session. None of the pictures used were part of any of the study lists (neither the picture itself nor the depicted concept). If the filler task would eliminate the effect, the color priming effect found in Experiment 1A would have to be interpreted as a very short-lived priming phenomenon.

2. On each test trial, four item versions were presented simultaneously (i.e., a red, blue, yellow, and green version of the drawing). The target color was presented equally often in any of the four positions of a $2 \times 2$ grid. Additionally, the positions of the four colors were changed from trial to trial (e.g., a red-colored drawing may have appeared in the upper left corner of the grid in the first trial, in the lower right corner in the second trial, in the lower left corner in the third trial, and in the upper right corner in the fourth trial). Every color appeared equally often in each of the four positions.

\section{Results}

In the preference test, target colors were chosen more often for old than for new item pairs $(M=31.0 \%, S E=8.9 \%$ versus $M=25.6 \%, S E=8.9 \%$, respectively, $t_{1}(47)=$ $\left.3.83, p_{1}<.01, t_{2}(63)=3.21, p_{2}<.01\right]$. In order to test how stable this effect was across subjects, we artificially split the total number of participants in half and repeated the analysis for each group. The split was based on workstation - that is, group 1 consisted of participants who were tested on workstations 1 and 2, and group 2 consisted of those tested on the other two workstations. In both groups, target colors were more often selected for old than for new items $\left[t_{1}(23) \geq 2.54, p_{1}<.01\right.$, one-tailed]. Priming scores (calculated as mean numbers of target colors selected to old items minus mean numbers of target colors selected to new items) did not differ between groups $(\mathrm{M}=5.2 \%$ versus $\left.M=5.6 \%, t_{1}<1\right)$.

Additionally, participants responded faster to old items $(M=1.27 \mathrm{~s}, S E=.21 \mathrm{~s})$ than to new items $[M=1.46 \mathrm{~s}$, $S E=.31 s, t_{1}(25)=3.49, p_{1}<.01, t_{2}(63)=4.41, p_{2}$ $<.01]$.

\section{Discussion}

The results of Experiment 1B closely resemble those of Experiment 1A: We detected a significant color priming effect. Target colors were selected more often for old than for new items, and participants processed old information faster than new information, as indicated by faster response times for old compared with new items. The priming effect does not appear to be overly short-lived because the filler task between study and test did not eliminate it. Although the priming effects in both experiments were rather low $(5$ to $7 \%$ ), they were nevertheless reliable. Additionally, other studies have reported similar effect sizes in mere exposure designs (e.g., Whittlesea \& Price, 2001). With the two parts taken together, Experiment 1 shows that significant and reliable color priming effects can be found in preference tests.

Is it safe, however, to interpret these priming effects as perceptual implicit memory effects? To address this question, we contrasted the preference test with a color-choice test in Experiment 2. The color-choice test was introduced by Wippich et al. (1994) and is assumed to rely mainly on conceptual implicit memory processes (see also Mecklenbräuker, Hupbach, \& Wippich, 2001, but also see Zimmer, Steiner, \& Ecker, 2002, for an alternative interpretation). During encoding, Wippich et al. and Mecklenbräuker et al. presented participants with colored line drawings. Following study, participants either were presented with blackand-white versions of studied and unstudied drawings or were given the names of previously studied and unstudied objects depicted in the drawings. All participants were asked to select the subjectively most appropriate color for each object depicted or referred to by the object's name. In both studies, significant effects of color priming were observed: The target color was selected more frequently for old items than for new items. More importantly, changing the stimulus presentation mode between study and test (from drawings to words) did not diminish priming. Because there was no overlap in perceptual attributes between study (colored drawings) and test items in the word condition, this finding indicates that the color-choice test mainly draws on conceptual processes and can thus be classified as a conceptual test of implicit memory (cf. Roediger, 1990).

In contrast to the color-choice test, the preference test used in the present study is assumed to rely mainly on perceptual processes or, according to the systems account of memory, to depend on the Structural Description System (e.g., Seamon et al., 1997, and Seamon, Williams, \& Crowley, 1995, but see Whittlesea \& Price, 2001, and Lawson, 2004, for alternative explanations of the mere exposure effect). If the preference test can indeed be classified as a perceptual implicit test, then variables that alter the global 
object shape should impair priming effects. However, as long as the identity of the object is preserved, these alterations should leave implicit effects in the color-choice test unaffected. Experiment 2 tested this hypothesis.

\section{Experiment 2}

Performance in conceptual implicit memory tests might be dissociated from performance in perceptual implicit tests by presenting different exemplars of the same concepts at test (e.g., Cave et al., 1996). For example, a table that is presented in the study session with a round top could be presented in the test session with the same or a differently shaped top. Presenting an alternative pictorial version of the same semantic concept (i.e., a table) should affect perceptual priming effects, but should not diminish priming in a conceptual implicit test, because only the physical attributes, and not the conceptual qualities, of the stimuli have changed from study to test. In Experiment 2, all items were presented as line drawings. Half of the studied items were presented as different exemplars of the same concepts at test, whereas for the other half of the items, identical exemplars were repeated at test. It was hypothesized that this variation would negatively affect only color priming in the preference test, but not color priming in the colorchoice test.

\section{Method}

\section{Design and Participants}

The experiment followed a $2 \times 3$ mixed factorial design. The between-subjects variable was test. Participants were randomly allocated to either the preference test or the color-choice test. Item status (same exemplars versus different exemplars versus new items) was varied within subjects.

Forty-eight adults participated in the experiment. The participants were undergraduate students at the University of Trier ( 30 females, 18 males; mean age $=23$ years; range $=18$ years to 35 years) who received course credit for their participation.

\section{Material}

Three lists of 20 line drawings were constructed. Participants received two lists (study lists) during the study session. The remaining list served as a new list at test. For one of the study lists, the same exemplars were repeated at test (same-exemplar list). For the other study list, different exemplars of the objects were presented at test (differentexemplar list). Item lists were counterbalanced (Latin square) throughout the experiment. Thus, across participants, each list served equally often as a same-exemplar, a different-exemplar, and a new list. During study, one item was shown at a time. There were five items for each of the four colors within each list. The colors were randomly allocated to the objects. Besides the additional presentation of different exemplars, the preference test was identical to that used in Experiment 1B (i.e., always four differently colored copies of one drawing were shown at a time). In the color-choice test, black-and-white versions of the drawings were presented one at a time (white outlines on a black computer screen). Four colored boxes (a blue-, a green-, a red-, and a yellow-filled rectangle) were presented side by side below the presented line drawings. These boxes served as buttons for participants' color selection. The serial order of the boxes was changed from trial to trial.

\section{Procedure}

The study session was identical to Experiment 1. Because there were no fundamental differences in the results between Experiments 1A and 1B, the filler task was abandoned in Experiment 2. After study, half of the participants received the color-choice test, and the other half received the preference test. The procedure for the preference test was identical to Experiment 1B. For the color-choice test, participants were asked to select the color that fitted the object best for each black-and-white item by clicking on one of the four colored boxes.

\section{Results}

Data sets from two participants (one in the color-choice and one in the preference test) had to be excluded from the analysis because of software malfunction. Hence, the final sample size was 46 participants.

First, we analyzed the baseline performances (preferences/choices for new items) and found no difference between the two tests $[M=25.9 \%, S E=8.9 \%$ for the preference, and $M=22.2 \%, S E=9.8 \%$ for the color-choice test, $t_{1}(44)=1.34, p_{1}=.19, t_{2}(59)=1.59, p_{2}=.12$, two-tailed]. In a second step, we calculated priming scores for same and different exemplars ( priming $_{\text {same }}=$ mean percentage of target-color selections for same exemplars $m i$ nus mean percentage of target-color selections for new items, priming $g_{\text {different }}=$ mean percentage of target-color selections for different exemplars minus mean percentage of target-color selections for new items; for mean scores see Figure 2).

A 2 (test: preference versus color-choice) $\times 2$ (exemplar type: same versus different) analysis of variance (ANOVA) performed on the priming scores showed significant effects of test $\left[F_{1}(1,44)=6.36, M S E_{1}=253.43, p_{1}=.02, F_{2}(1\right.$, $\left.59)=6.90, M S E_{2}=627.82, p_{2}=.01\right]$ and exemplar type $\left[F_{1}(1,44)=7.89, M S E_{1}=104.47, p_{1}<.01, F_{2}(1,59)=\right.$ 7.13, $\left.M S E_{2}=311.84, p_{2}=.01\right]$. Priming effects in the color-choice test $(M=11.8 \%, S E=10.5 \%)$ were higher than priming scores in the preference test $(M=3.4 \%, S E$ $=12.0 \%)$. Modified pictures $(M=4.6 \%, S E=13.0 \%)$ led to less priming than identical pictures $(M=10.6 \%, S E$ $=14.8 \%)$. The interaction did not reach significance $\left[F_{1}(1\right.$, $44)=1.38, M S E_{1}=104.47, p_{1}=.25, F_{2}(1,59)=1.55$, $\left.M S E_{2}=274.03, p_{2}=.22\right]$.

Separate $t$-tests showed that the priming score for different exemplars in the preference test did not significantly differ from zero $\left[t_{1}(22)=-.36, p_{1}=.73, t_{2}(59)=-.42\right.$, $\left.p_{2}=.68\right]$. All other priming scores were above chance level $[t \geq 2.21, p \leq .03]$. 


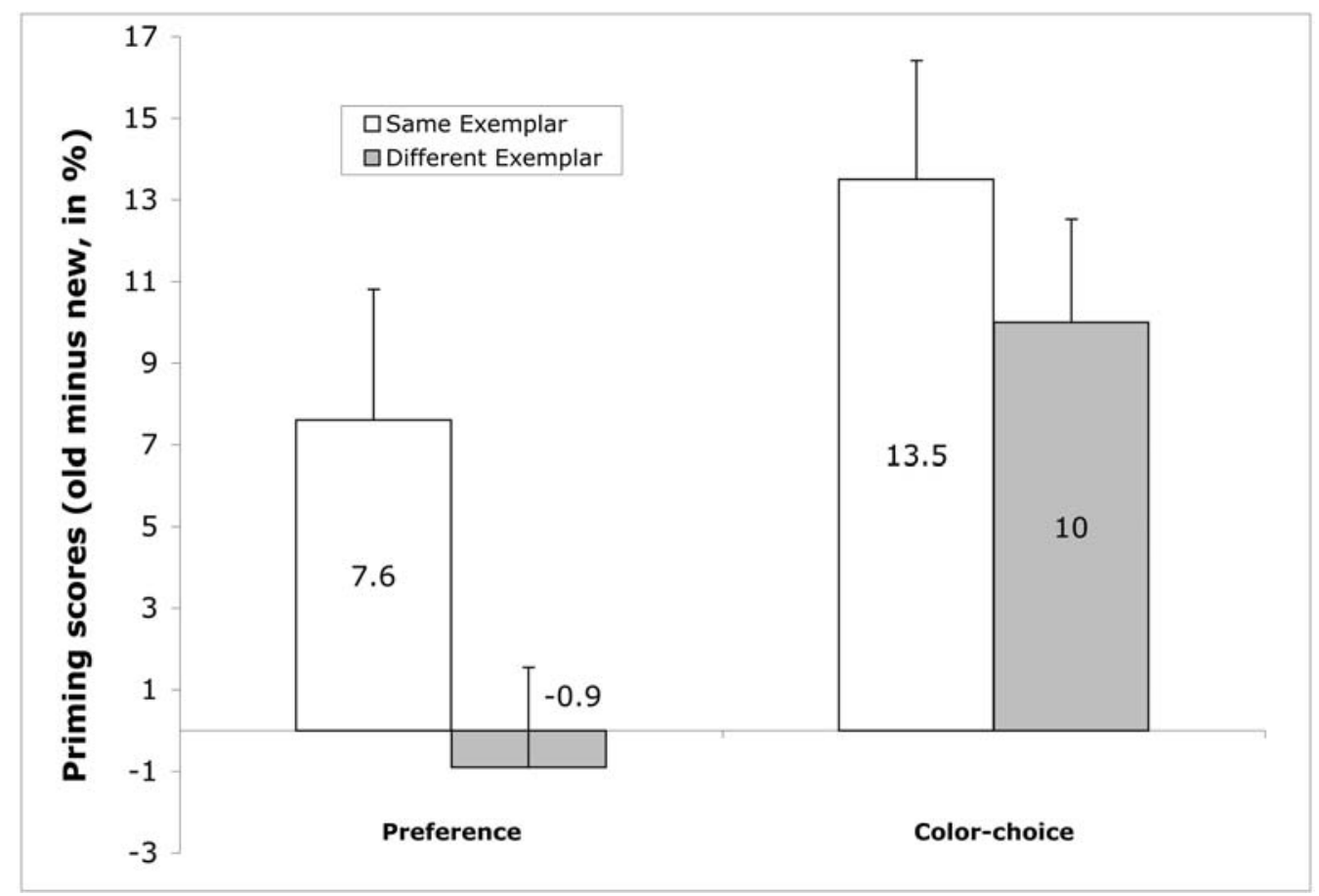

Figure 2. Priming effects (old minus new, in \%) as a function of exemplar type (same versus different) in the color-choice and the preference test in Experiment 2. (Error bars represent standard errors of means.)

Additionally, we analyzed the mean response times with a 2 (test: preference versus color-choice) $\times 3$ (exemplar type: same versus different versus new) ANOVA. Surprisingly, and in contrast with Experiments $1 \mathrm{~A}$ and $1 \mathrm{~B}$, the byparticipant analysis did not reveal any significant effects $(F \leq 2.24)$. The by-item analysis revealed a marginally significant effect of status $\left[F_{2}(2,118)=2.43, M S E_{2}=\right.$ $\left..06, p_{2}=.09\right]$.

\section{Discussion}

The analysis of the number of target colors selected to old and new items again revealed significant color priming effects in the implicit preference test. Most importantly, the results of Experiment 2 support the view that the preference test can be classified as a perceptual implicit test: significant priming effects were only observed when the object shape was preserved (same exemplars). We did not find color priming when there was only a conceptual match between items in the study and test session (different exemplars). This effect has to be interpreted with caution because it was not very robust (the interaction of the $2 \times 2$ ANOVA performed on the priming scores did not reach significance). However, the priming effect for modified pictures in the preference test was even slightly negative. Apparently, color priming depends on the repetition of the perceptual attributes of the study items at test. Reactivating the conceptual representations of the studied items was insufficient to reveal color priming in the preference test. In contrast, we found significant priming for same and different exemplars in the color-choice test. The numerical advantage of same over different exemplars in the color- choice test can be interpreted as a perceptual component of an otherwise conceptually driven implicit test (e.g., Craik et al., 1994; Weldon, 1991). Alternatively, as suggested by an anonymous reviewer, it could be argued that both implicit tests are similarly influenced by changes of shape information, but that the color-choice test has an additional shape-independent component.

Experiment 2 extends the results reported by Wippich et al. (1994) and Mecklenbräuker et al. (2001) by showing that the color-choice test is not substantially affected by changes of the stimulus shape. This supports the view that the color-choice test can be classified as an implicit memory test that largely depends on conceptual processing (but see Zimmer et al., 2002).

\section{Experiment 3}

The purpose of Experiment 3 was to test the possibility that implicit memory performances in Experiment 2 were contaminated by explicit retrieval attempts. Experiments 1 and 2 did not allow estimations of the participants' ability to explicitly remember the colors of the presented stimuli. Therefore, it remains unclear whether the participants tried to remember the study colors explicitly instead of spontaneously choosing the preferred color version (preference test) or selecting the most appropriate color (color-choice test). Although it seems rather unlikely that participants voluntarily used this more effortful strategy, we cannot rule out this possibility unless we find dissociations between implicit and explicit test versions. Experiment 3 intended to compare the implicit tests used in Experiment 2 with corresponding explicit test versions. In Experiment 3 we 
administered explicit tests that differed from the implicit tests of Experiment 2 only in their test instructions. This fulfills the retrieval intentionality criterion of Schacter, Bowers, and Booker (1989). According to this criterion, an explicit contamination of the implicit test can be ruled out if an experimental variable is demonstrated to dissociate the implicit from the explicit memory test, provided that the same nominal cues are used in both tests and that the tests only vary in their instructions (implicit versus explicit). Schacter et al. (1989) argued that the dissociative effect of the variable can then be attributed to the differences in the unintentional and intentional retrieval processes that are used to perform the tests.

The independent variable that was varied in Experiments 2 and 3 was the exemplar version. Studied objects were presented as either same or different exemplars. Based on the perceptual-conceptual processing framework, we hypothesized that an exemplar change should affect only the implicit preference test, because this test largely depends on perceptual processes. In contrast, both of the explicit tests and the implicit color-choice test should not be affected by exemplar change because of the conceptual nature of these tests.

However, possible dissociations between implicit and explicit tasks have to be interpreted with caution because explicit tasks are often more reliable than implicit tasks (Buchner \& Brandt, 2003; Buchner \& Wippich, 2000; Meier \& Perrig, 2000). Therefore, explicit tasks often have more statistical power to detect possible differences caused by an independent variable.

\section{Method}

\section{Design and Participants}

A $2 \times 2$ mixed factorial design was used in Experiment 3 , with explicit test serving as a between-subjects variable and exemplar version (same vs. different) serving as a within-subjects variable. Half of the participants were randomly allocated to the explicit version of the preference test; the other half received the explicit version of the colorchoice test.

Forty-eight adults participated in the experiment. The participants were undergraduate students at the University of Arizona ( 27 females, 21 males; mean age $=20$ years; range $=18$ years to 30 years) who received course credit for their participation.

\section{Material and Procedure}

The same material as in Experiment 2 was used except that only two lists were presented because no new items were included in the explicit tests. The procedure also closely resembled Experiment 2 with the exception that participants were asked to explicitly remember the colors of the items from the study session regardless of the particular exemplar shown (same versus different). Participants were asked to make their decisions either by clicking directly on one of the four color versions of the drawing (explicit preference test) or by clicking on one of the color boxes presented below the black-and-white versions of the drawings (explicit color-choice test).

\section{Results}

Percentages of remembered target colors were submitted to a 2 (explicit test: preference versus color-choice) $\times 2$ (exemplar type: same versus different) ANOVA, which revealed that more target colors were remembered in the explicit color-choice test than in the explicit version of the preference test $\left[F_{1}(1,46)=11.15, M S E_{1}=298.31, p_{1}=\right.$ $\left..02 ; \mathrm{F}_{2}(1,39)=28.83, \mathrm{MSE}_{2}=192.21, p_{2}<.01\right]$ and that more target colors were remembered for items that were presented in the same pictorial format than for items that were presented as different exemplars $\left[F_{1}(1,46)=23.39\right.$, $M S E_{1}=147.27, p_{1}<.01 ; F_{2}(1,39)=25.77, M S E_{2}=$ $222.75, p_{2}<.01$; for means see Figure $3 ; \mathrm{F}<1$ for the interaction]. However, colors were remembered well above chance level $(25 \%)$ for different exemplars in both tests $\left[t_{1}(23) \geq 12.9, p_{1}<.01, t_{2}(39) \geq 10.4, p_{2}<.01\right]$.

In order to see whether participants responded differently in the implicit and explicit tests, we compared the mean response times in Experiments 2 and 3 with a 2 (test: preference versus color-choice) $\times 2$ (version: implicit versus explicit) $\times 2$ (exemplar type: same versus different) mixed ANOVA. We found main effects of version $\left[F_{1}(1\right.$, $90)=85.15, M S E_{1}=1.03, p_{1}<.01 ; F_{2}(1,39)=270.04$, $\left.M S E_{2}=.56, p_{2}<.01\right]$ and exemplar type $\left[F_{1}(1,90)=\right.$ $42.90, M S E_{1}=.19, p_{1}<.01 ; F_{2}(1,39)=28.36, M S E_{2}$ $\left.=.43, p_{2}<.01\right]$ and a significant version by exemplar type interaction $\left[F_{1}(1,90)=29.78, M S E_{1}=.19, p_{1}<\right.$ $.01 ; F_{2}(1,39)=22.30, M S E_{2}=.44, p_{2}<.01$; all other $F \mathrm{~s} \leq 1.84]$. Analyses of the simple effects showed that participants responded equally fast to same and different exemplars in the implicit tests $[\mathrm{F} \leq 1.32]$. In contrast, participants responded significantly faster to same than to different exemplars in the explicit tests $\left[F_{1}(1,92)=75.05\right.$, $M S E_{1}=.19, p_{1}<.01 ; F_{2}(1,39)=26.87, M S E_{2}=.81$, $\left.p_{2}<.01\right]$. Additionally, response times were slower in the explicit than in the implicit tests for both same $\left[F_{1}(1,90)\right.$ $=63.14, M S E_{1}=.39, p_{1}<.01 ; F_{2}(1,39)=123.74, M S E_{2}$ $\left.=.34, p_{2}<.01\right]$ and different exemplars $\left[F_{1}(1,90)=\right.$ 82.77, $M S E_{1}=.84, p_{1}<.01 ; F_{2}(1,39)=181.06, M S E_{2}$ $\left.=.66, p_{2}<.01\right]$.

\section{Discussion}

The explicit color-choice test led to better memory performance than the explicit preference test. This finding likely reflects differences in the cognitive demands imposed by the tasks. Participants in the color-choice test were presented with only one black-and-white drawing at a time. Thus, the color-choice test can roughly be classified as a cued recall test, with the presented black-and white drawing serving as a retrieval cue for the color feature. In contrast, the explicit preference test was presented as a forcedchoice recognition test with four drawings presented at once that differed only in color. It is known that recognition performance depends on the degree of similarity between targets and distractors (e.g., Ley \& Long, 1988). The test format used in the explicit preference test might have made it especially difficult for the participants to remember the correct color; targets and distractors were highly similar. 


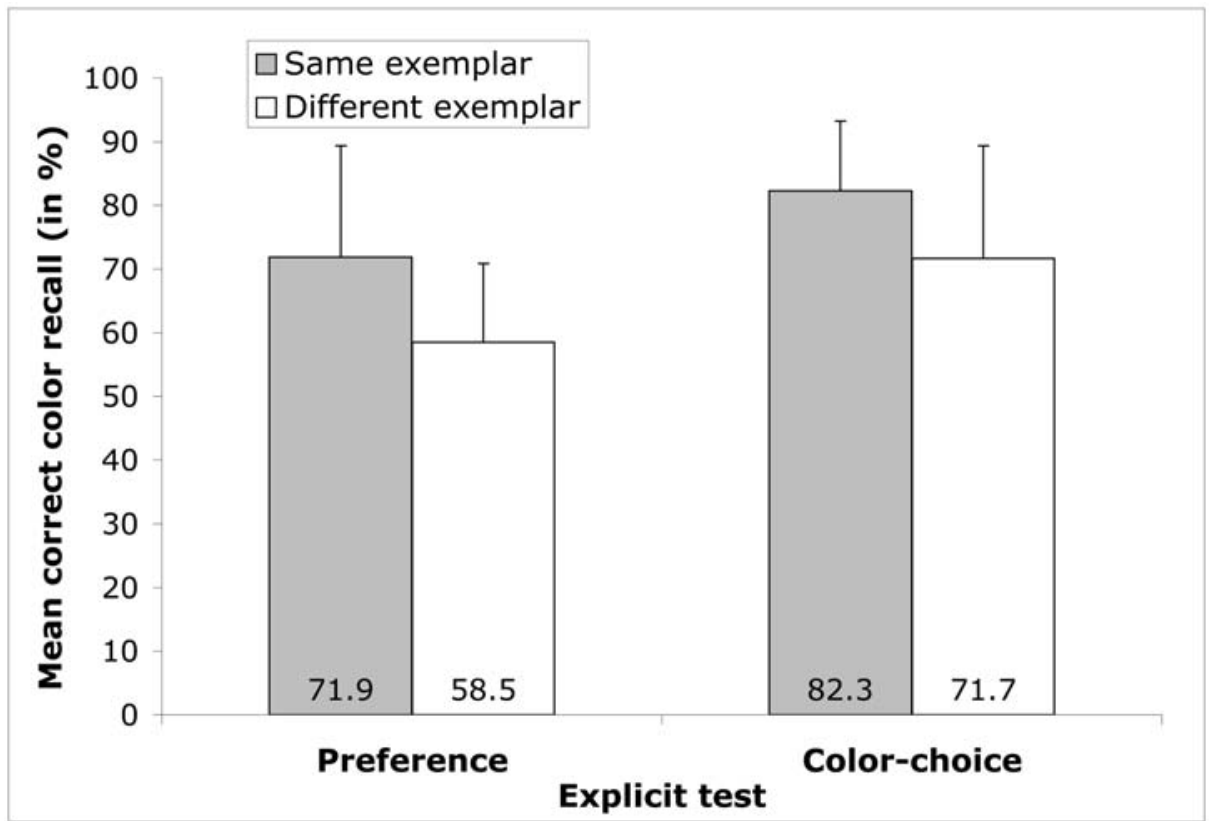

Figure 3. Number of target colors (in \%) correctly remembered as a function of exemplar type (same versus different) and test (explicit color-choice versus explicit preference test) in Experiment 3. (Error bars represent standard errors of means.)

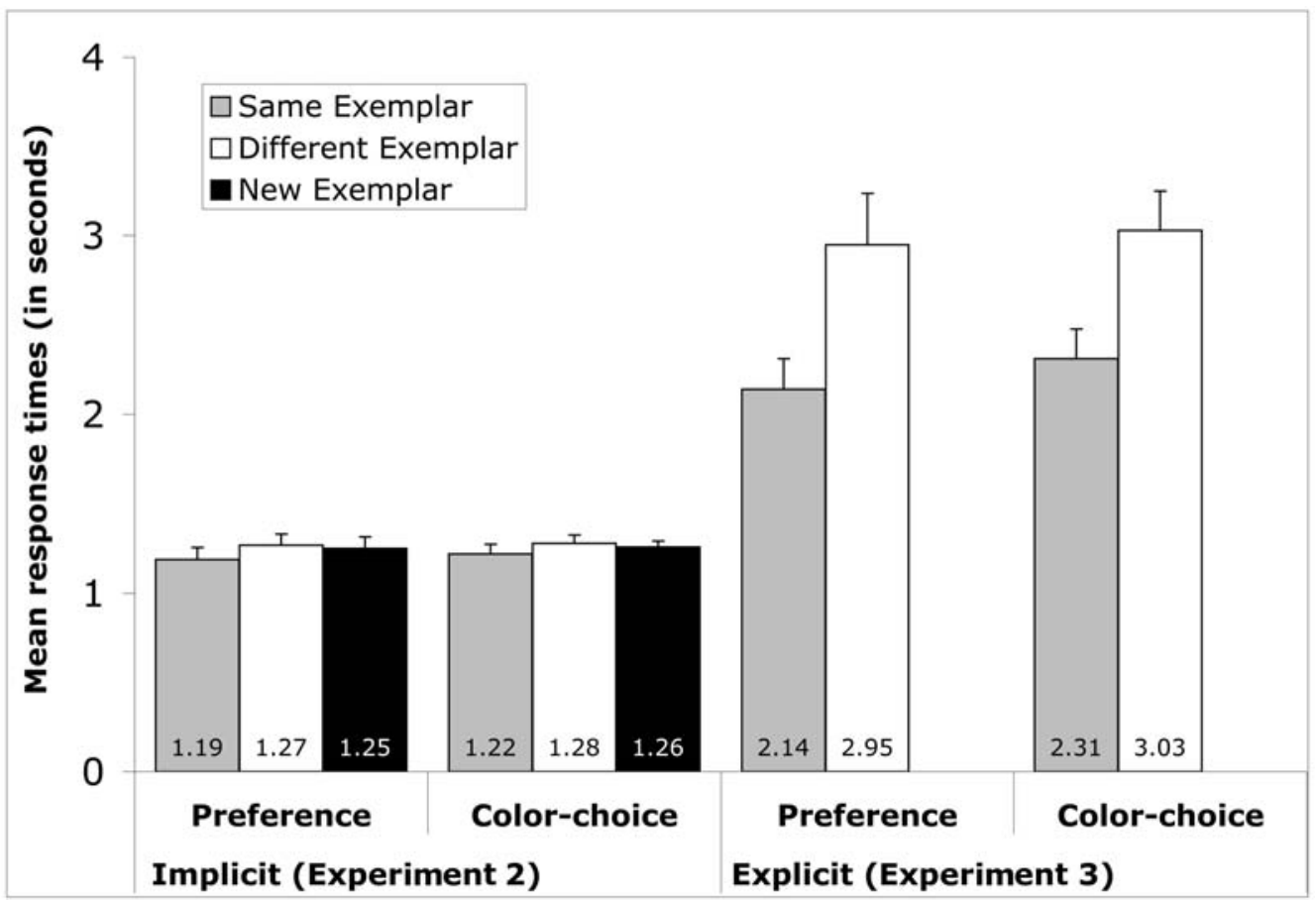

Figure 4. Mean response times (in seconds) in the implicit (Experiment 2) and explicit tests (Experiment 3) as a function of exemplar type (same versus different) and test (color-choice versus preference test). (Error bars represent standard errors of means.)

In both explicit tests in Experiment 3, memory for colors benefited from the preservation of the object shape, an unexpected finding that was mirrored in the response times (see later discussion). This result speaks for a strong perceptual component of both explicit tests. Blaxton (1989) already demonstrated that explicit tests can be driven by perceptual processes. We nevertheless tend to classify the explicit tests in the present study as mainly conceptually driven because participants (a) were informed that some of the presented exemplars were different from those seen 
during study and (b) were instructed to ignore the perceptual changes and to remember the colors in which the concepts were originally presented. In general, there has been considerable uncertainty regarding the perceptual specificity of explicit tests (cf. Rajaram, 1996; Reder, Donavos, \& Erickson, 2002). Although some researchers did not find effects of perceptual similarity between study and test items in explicit memory (e.g., Craik et al., 1994; Rajaram \& Roediger, 1993), others reported such effects (e.g., Graf \& Ryan, 1990; Rajaram, 1996; Reder et al., 2002; see Zimmer \& Steiner, 2003, for color specificity in episodic object memory and Engelkamp, Zimmer, \& DeVega, 2001, for a recent review on the influence of perceptual qualities on episodic object memory). Although the perceptual-conceptual processing account has difficulties explaining these differences, recent accounts that are based on Hunt and colleagues' (e.g., Hunt \& McDaniel, 1993) notion of the positive effects of distinctiveness on remembering offer theoretical alternatives (see, for example, Rajaram, 1996, and Reder et al., 2002, for detailed empirical and theoretical considerations).

Participants responded much slower in the explicit than in the implicit tests. This suggests that they intentionally tried to remember the colors in the explicit tests, whereas they spontaneously chose one of the four colors in the implicit tests.

The implicit tests used in Experiment 2 differed from the explicit tests used in Experiment 3 only in the test instructions (the only other difference is that new items were only presented in the implicit test and not in the explicit test version). Therefore, the retrieval intentionality principle is met, which permits us to explain dissociations between the implicit and the explicit tests as a function of different processes underlying implicit and explicit test performance. However, dissociations have to be interpreted with caution because of the possibility that the explicit and the implicit test differ in their reliability (Buchner \& Brandt, 2003; Buchner \& Wippich, 2000; Meier \& Perrig, 2000). Although the exemplar change negatively affected the number of target colors chosen in both the implicit and explicit preference test, changing the exemplars eliminated color priming in the implicit preference test whereas in the explicit preference test participants still remembered the colors of different exemplars well above chance levels. Additionally, the exemplar change differently affected the response times in the implicit and explicit tests. Participants responded equally quickly to the varied exemplar types (same, different, new) in the implicit tests. In contrast, it took them longer to remember the colors for changed than for unchanged exemplars in the explicit tests. This finding speaks against an explicit contamination of the implicit test performances as well as against an implicit contamination of the explicit test performance. If participants had been trying to remember explicitly instead of choosing spontaneously the colors in the implicit tests, they should have shown a much better memory performance in the implicit tests, and they should have remembered the colors of changed exemplars well above chance level in the preference test. Additionally, participants should have responded more slowly and differently to same and different exemplars in the implicit tests.
Do the reported differences between the implicit and the explicit version of the preference test mean that each version operated upon a different representational system? Although effects in perceptual implicit tests are usually attributed to the PRS, Lawson (2004) and Zimmer et al. (2002) have challenged this view. With regard to implicit color effects, Zimmer et al. argue that these effects are caused by an automatic retrieval of an episodic token, which is also responsible for explicit memory effects. The episodic token is assumed to integrate all features, including perceptual ones that are processed during encoding. For the color-choice test, Zimmer et al. showed that performance was not independent of explicit recollection. This does not mean that participants tried to explicitly remember the color during the implicit test. Rather, participants involuntarily and automatically activated the same episodic memory traces that are used in explicit tests. Future studies are needed to clarify whether the same interpretation applies to the color-preference test that was used in the present study.

\section{General Discussion}

Almost every study on implicit color effects used objectidentification paradigms, such as object naming or picture fragment identification. These studies consistently showed that changes of color, location, size, or object orientation do not affect priming. Object identification is mainly based on the analyses of the object shape, and given normal acuity, object shape is accessible regardless of changes in color, size, location, and orientation. Cave et al. (1996) suggested that only modifications that alter the object shape influence priming effects in identification paradigms. However, as noted by Wippich et al. (1994), it would be premature to conclude that the specific perceptual attributes are not part of the representational system underlying perceptual priming effects. The present study shows that although perceptual priming processes can work color-independently, color information can nevertheless be accessed by perceptual implicit tests.

To our knowledge, the present study is the first demonstration of color effects in a mere exposure design. Unlike previous studies, the test task in the present study did not require object identification or other shape-based object evaluations. Instead, a preference test was used in which participants always had to choose between differently colored drawings of the same object. Therefore, test performance was solely based on color information. The perceptual nature of the preference test is corroborated by Experiment 2, which showed that altering the object shape by presenting a different exemplar of the same-named concept eliminated priming effects in this test. In an overall analysis of the priming scores, the preference and the colorchoice test were affected similarly by the exemplar change. However, changing the exemplar affected the color-choice test much less than the preference test: priming effects for both same and different exemplars reached significance in the color-choice test. The results of Experiment 3 led to the conclusion that the implicit effects found in Experi- 
ments 1 and 2 were not an artifact of explicit retrieval attempts.

In the present article, we have focussed on the retrieval situation and the perceptual and conceptual mechanisms of memory for color information. We have not yet addressed the role of the encoding situation, which provides the basis for implicit and explicit memory for object colors. In visual perception, feature binding denotes the process of establishing an integrated representation of the object's visual properties (e.g., Treisman, 1999). Binding provides the memorial experience that certain features belong together (Chalfonte \& Johnson, 1996). Binding, however, is a deliberate strategic act that requires focal attention to stimulus features (Treisman \& Gelade, 1980). Preventing people from focussing attention (e.g., by giving only brief presentations) typically results in binding errors - people erroneously put features together by recombining the shape, size, format, and color (Treisman \& Schmidt, 1982).

In addition to feature binding, it is important to note that object features differ in terms of the amount of cognitive effort required for their encoding. Unlike other object features (e.g., spatiotemporal context), color is not automatically encoded together with object identity but requires controlled processing (Park \& Mason, 1982; see Hasher \& Zacks, 1979, for the distinction between automatic and effortful processes). Hence, recalling the nondiagnostic (i.e., arbitrarily chosen) color of an object requires that color and form information have been bound together in a deliberate strategic act that requires focal attention during encoding (Hanna \& Remington, 1996). Evidence for this view also comes from studies in the explicit memory domain: memory for object color was shown to benefit from binding of color and shape information during encoding (Chalfonte \& Johnson, 1996; Park \& Mason, 1982).

The literature on implicit memory suggests that, during encoding, allocating attention to color might also be necessary for color priming. Musen and O’Neill (1997) reported that color priming depended on whether attention was paid to the color attribute during encoding. Color effects were found in an implicit color-naming test when the encoding task encouraged the unitization between color and shape information by asking participants to form an image of the shape in the presented color. At test, colors of shapes that were unchanged from study to test were named faster than colors of shapes that appeared in new combinations of colors and shapes. In addition, Musen et al. (1999) reported that color priming was not confined to study conditions in which objects and colors were already physically integrated (i.e., colored objects). In their study, the physical separation of features was overcome by encoding conditions that required the participants to pay attention both to objects (i.e., words) and colors. This was done by having the participants form mental color images of the objects that were printed in black according to the color of the surrounding background frame. Musen et al. (1999) reasoned that color priming depended on the fact that color information and object identity had been unitized into a single item during the study trial (see also Mecklenbräuker et al., 2001).

Recent studies by Lloyd-Jones (2005) and Vernon and
Lloyd-Jones (2003) followed Musen et al. (1999) to the effect that color priming is thought to reflect a degree of new-association priming. Yet the exact conditions under which new associations between perceptual features occur are not fully understood. Whereas Musen and her colleagues $(1997,1999)$ argued that color priming requires focal attention during encoding, Lloyd-Jones (2005) and Vernon and Lloyd-Jones (2003) found equivalent color priming for both colored-object decision and object naming as study tasks. Colored-object decisions clearly require participants to attend to both objects and colors (which is also the case with the color-object ratings in the present experiments). In contrast, color was not relevant for the naming task. Nevertheless, this study task led to significant color effects. The question of whether implicit color effects are limited to conditions in which attention is explicitly drawn to color information during encoding or whether color is encoded automatically has to await further research.

Srinivas's (1996) finding of size effects and the present study on color effects show that specific object attributes can be "remembered" implicitly when study and test tasks are used that activate processes that are sensitive to these object attributes. However, because of the possible constraints set by the PRS, it is not guaranteed that using the "right" task will lead to priming effects. Although color and size information seem to be represented in the system, left-right orientation seems not to be included (Srinivas, 1996). Further research is needed to reveal the specific constraints of the system(s) mediating perceptual priming. It is important to note that in Srinivas's study and in our study the consequent implementation of the TAP principle challenged previous assumptions about implicit color effects. Therefore, we believe that processing accounts of implicit memory seem to be especially fruitful when it comes to finding experimental conditions that critically test current assumptions (e.g., Moscovitch, 1992, 1994; Ecker, GrohBordin, \& Zimmer, 2004) about the way memories are represented in the brain.

\section{Acknowledgments}

We would like to thank Toby Lloyd-Jones and an anonymous reviewer for their helpful comments on an earlier draft of our paper. We would also like to thank Silvia Mecklenbräuker and Werner Wippich for their continuous support of our research.

\section{References}

Biederman, I., \& Ju, G. (1988). Surface versus edge-based determinants of visual recognition. Cognitive Psychology, 20, 38 64.

Blaxton, T. A. (1989). Investigating dissociations among memory measures: Support for a transfer-appropriate processing framework. Journal of Experimental Psychology: Learning, Memory \& Cognition, 15, 657-668.

Buchner, A., \& Brandt, M. (2003). Further evidence for systematic reliability differences between explicit and implicit memory tests. Quarterly Journal of Experimental Psychology: Human Experimental Psychology, 56(2), 193-209. 
Buchner, A., \& Wippich, W. (2000). On the reliability of implicit and explicit memory measures. Cognitive Psychology, 40(3), 227-259.

Cave, C. B., Bost, P. R., \& Cobb, R. E. (1996). Effects of color and pattern on implicit and explicit picture memory. Journal of Experimental Psychology: Learning, Memory, \& Cognition, 22, 639-653.

Chalfonte, B. L., \& Johnson, M. K. (1996). Feature memory and binding in young and older adults. Memory \& Cognition, 24, 403-416.

Cooper, L. A., Schacter, D. L., Ballesteros, S., \& Moore, C. (1992). Priming and recognition of transferred three dimensional objects: Effects of size and reflectance. Journal of Experimental Psychology: Learning, Memory, \& Cognition, 18, 43-57.

Craik, F.I.M., Moscovitch, M., \& McDowd, J. M. (1994). Contributions of surface and conceptual information to performance on implicit and explicit memory tasks. Journal of Experimental Psychology: Learning, Memory, \& Cognition, 20, 864-875.

Ecker, U.K.H., Groh-Bordin, C., \& Zimmer, H. D. (2004). Electrophysiological correlates of specific feature binding in remembering-Introducing a neurocognitive model of human memory. In A. Mecklinger, H. D. Zimmer, \& U. Lindenberger (Eds.), Bound in Memory (pp. 179-193). Aachen, Germany: Shaker Verlag.

Engelkamp, J., Zimmer, H. D., \& de Vega, M. (2001). Pictures and words in memory: The role of visual-imaginal information. In M. Dennis, C. Cornoldi, R. H. Logie, M. DeVega, \& J. Engelkamp (Eds.), Imagery, Language and Visuo-Spatial Thinking (pp. 59-80). Hove, UK: Psychology Press.

Graf, P., \& Ryan, L. (1990). Transfer-appropriate processing for implicit and explicit memory. Journal of Experimental Psychology: Learning, Memory, \& Cognition, 16, 978-992.

Hanna, A., \& Remington, R. (1996). The representation of color and form in long-term memory. Memory \& Cognition, 24, 322-330.

Hasher, L., \& Zacks, R. T. (1979). Automatic and effortful processes in memory. Journal of Experimental Psychology: General, 108, 356-388.

Hunt, R. R., \& McDaniel, M. A. (1993). The enigma of organization and distinctiveness. Journal of Memory \& Language, 32, 421-445.

Jacoby, L. L., \& Dallas, M. (1981). On the relationship between autobiographical memory and perceptual learning. Journal of Experimental Psychology: General, 110, 306-340.

Lawson, R. (2004). Depth rotation and mirror-image reflection reduce affective preference as well as recognition memory for pictures of novel objects. Memory \& Cognition, 32(7), 11701181.

Ley, R., \& Long, K. (1988). Distractor similarity effects in tests of discrimination recognition and distractor-free recognition. Bulletin of the Psychonomic Society, 26, 407-409.

Lloyd-Jones, T. (2005). The role of colour in the implicit memory performance of healthy older adults and individuals with Alzheimer's disease. Neuropsychology, 19, 44-53.

Mecklenbräuker, S., Hupbach, A., \& Wippich, W. (2001). What color is the car? Implicit memory for color information in children. Quarterly Journal of Experimental Psychology: Human Experimental Psychology, 54, 1069-1086.

Meier, B., \& Perrig, W. J. (2000). Low reliability of perceptual priming: Consequences for the interpretation of functional dis- sociations between explicit and implicit memory. Quarterly Journal of Experimental Psychology: Human Experimental Psychology, 53, 211-233.

Moscovitch, M. (1992). Memory and working-with-memory: A component process model based on modules and central systems. Journal of Cognitive Neuroscience, 4, 257-267.

Moscovitch, M. (1994). Cognitive resources and DA interference effects at retrieval in normal people: The role of the frontal lobes and medial temporal cortex. Neuropsychology, 8, 524534.

Musen, G., \& O’Neill, J. E. (1997). Implicit memory for nonverbal associations. Journal of Experimental Psychology: Learning, Memory, \& Cognition, 23, 1192-1202.

Musen, G., Szerlip, J. S., \& Szerlip, N. J. (1999). Role of familiarity and unitization on new-association priming. Journal of Experimental Psychology: Learning, Memory, \& Cognition, 25, 275-283.

Park, D. C., \& Mason, D. (1982). Is there evidence for automatic processing of spatial and color attributes present in matched pictures and words? Memory \& Cognition, 10, 76-81.

Rajaram, S. (1996). Perceptual effects on remembering: Recollective processes in pictures recognition memory. Journal of Experimental Psychology: Learning, Memory, \& Cognition, 22, 365-377.

Rajaram, S., \& Roediger, H. L. (1993). Direct comparison of four implicit memory tasks. Journal of Experimental Psychology: Learning, Memory, \& Cognition, 19, 765-776.

Reder, L. M., Donavos, D. K., \& Erickson, M. A. (2002). Perceptual match effects in direct tests of memory: The role of contextual fan. Memory \& Cognition, 30, 312-323.

Roediger, H. L. (1990). Implicit memory: Retention without remembering. American Psychologist, 45, 1043-1056.

Roediger, H. L., \& Blaxton, T. A. (1987). Effects of varying modality, surface features and retention interval on priming in word-fragment completion. Memory \& Cognition, 15, 379388.

Roediger, H. L., \& McDermott, K. B. (1993). Implicit memory in normal human subjects. In F. Boller \& J. Grafman (Eds.), Handbook of Neuropsychology (Vol. 8, pp. 63-131). Amsterdam: Elsevier.

Roediger, H. L., Weldon, M. S., \& Challis, B. H. (1989). Explaining dissociations between implicit and explicit measures of retention: A processing account. In H. L. Roediger \& F.I.M. Craik (Eds.), Varieties of memory and consciousness: Essays in honour of Endel Tulving (pp. 3-41). Hillsdale, NJ: Lawrence Erlbaum.

Schacter, D. L. (1992). Understanding implicit memory: A cognitive neuroscience approach. American Psychologist, 47, 559-569.

Schacter, D.L. (1994). Priming and multiple memory system: Perceptual mechanisms of implicit memory. In D. L. Schacter \& E. Tulving (Eds.), Memory systems 1994 (pp. 233-268). Cambridge, MA: The MIT Press.

Schacter, D. L., Bowers, J., \& Booker, J. (1989). Intention, awareness, and implicit memory: The retrieval intentionality criterion. In S.Lewandowsky, J. C.Dunn, \& K.Kirsner (Eds.), Implicit memory: Theoretical issues (pp. 47-65). Hillsdale, NJ: Lawrence Erlbaum.

Seamon, J. G., \& Delgado, M. R., (1999). Recognition memory and affective preference for depth-rotated solid objects: Partbased structural descriptions may underlie the mere exposure effect. Visual Cognition, 6, 145-164. 
Seamon, J. G., Ganor-Stern, D., Crowley, M. J., Wilson, S. M., Weber, W. J., O'Rourke, C. M., \& Mahoney, J. K. (1997). A mere exposure effect for transformed three-dimensional objects: Effects of reflection, size, or color changes on affect and recognition. Memory \& Cognition, 25, 367-374.

Seamon, J. G., Williams, P. C., \& Crowley, M. J. (1995). The mere exposure effect is based on implicit memory: Effects of stimulus type, encoding conditions, and number of exposures on recognition and affect judgments. Journal of Experimental Psychology: Learning, Memory, \& Cognition, 21, 711-721.

Srinivas, K. (1993). Perceptual specificity in nonverbal priming. Journal of Experimental Psychology: Learning, Memory, \& Cognition, 19, 582-602.

Srinivas, K. (1996). Size and reflection effects in priming: A test of transfer-appropriate processing. Memory \& Cognition, 24, 441-452.

Treisman, A. (1992). Perceiving and re-perceiving objects. American Psychologist, 47, 862-875.

Treisman, A. (1999). Feature binding, attention and object perception. In G. W.Humphreys, J.Duncan, \& A.Treisman (Eds.), Attention, space and action. Studies in cognitive neuroscience (pp. 91-111). Oxford: Oxford University Press.

Treisman, A., \& Gelade, G. (1980). A feature-integration theory of attention. Cognitive Psychology, 12, 97-136.

Treisman, A., \& Schmidt, H. (1982). Illusory conjunctions in the perception of objects. Cognitive Psychology, 14, 107-141.

Vernon, D., \& Lloyd-Jones, T. J. (2003). The role of colour in implicit and explicit memory performance. The Quarterly Journal of Experimental Psychology, 56A(5), 779-802.

Weldon, M. S. (1991). Mechanisms underlying priming on per- ceptual tests. Journal of Experimental Psychology: Learning, Memory, \& Cognition, 175, 526-541.

Whittlesea, B.W.A., \& Price, J. R. (2001). Implicit/explicit memory versus analytic/ nonanalytic processing: Rethinking the mere exposure effect. Memory \& Cognition, 29, 234-246.

Wippich, W., \& Mecklenbräuker, S. (1998). Effects of color on perceptual and conceptual tests of implicit memory. Psychological Research, 61, 285-294.

Wippich, W., Mecklenbräuker, S., \& Baumann, R. (1994). Farbwirkungen bei impliziten und expliziten Gedächtnisprüfungen [The effects of color in implicit and explicit tests of memory]. Zeitschrift für experimentelle und angewandte Psychologie, 41, 315-347.

Zimmer, H. D., \& Steiner, A. (2003). Colour specificity in episodic and in perceptual object recognition with enhanced colour impact. European Journal of Cognitive Psychology, 15, 349-370.

Zimmer, H. D., Steiner, A., \& Ecker, U.K.H. (2002). How “implicit" are implicit color effects in memory? Experimental Psychology, 49, 120M-131.p

Almut Hupbach

Department of Psychology

University of Arizona

Tucson, AZ 85721

USA

E-mail ahupbach@email.arizona.edu

Tel. + 15206260278 